\title{
Effectiveness of ultrasound therapy for myofascial pain syndrome: a systematic review and meta-analysis
}

\author{
Peng Xia* \\ Xiaoju Wang* \\ Qiang Lin \\ Kai Cheng \\ Xueping Li
}

Department of Rehabilitation Medicine, Nanjing First Hospital, Nanjing Medical University, Nanjing, People's Republic of China

*These authors contributed equally to this work
Correspondence: Xueping Li

Department of Rehabilitation Medicine, Nanjing First Hospital, Nanjing Medical

University, 68 Changle Road, Nanjing

210006, People's Republic of China

$\mathrm{Tel}+86 \quad 18951670129$

Email lixueping6504@I63.com
This article was published in the following Dove Press journal: Journal of Pain Research

7 March 2017

Number of times this article has been viewed

Objective: The objective of this review was to assess the therapeutic effect of ultrasound (US) on myofascial pain syndrome (MPS).

Date sources: PubMed, Embase, and Cochrane Library were searched to find relevant studies from January 1966 to May 2016 using keywords. Four investigators performed the data extraction. Study selection: Randomized controlled trials (RCTs) investigating the outcomes of pain and physical function between MPS patients receiving and not receiving US were selected by two researchers independently.

Data extraction: Data were extracted from the RCTs. Risk of bias and study quality were evaluated following the recommendations of Cochrane Collaboration. Standardized mean difference (SMD) and 95\% confidence interval (CI) were calculated.

Data synthesis: A total of 10 studies involving 428 MPS patients were included. US therapy significantly reduced pain intensity (SMD $[\mathrm{CI}]=-1.41[-2.15,-0.67], P=0.0002)$ and increased pain threshold (SMD $[\mathrm{CI}]=1.08[0.55,1.60], P<0.0001)$, but had no significant effect on cervical range of motion $(\mathrm{ROM})$ of lateral flexion (SMD $[\mathrm{CI}]=0.40[-0.19,0.99], P=0.19)$, rotation (SMD $[\mathrm{CI}]=0.10[-0.33,0.52], P=0.66)$, or extension or flexion $(\mathrm{SMD}[\mathrm{CI}]=0.16[-0.35,0.68]$, $P=0.53$ ). Heterogeneity between studies was mainly attributed to differences in the follow-up time, parameter of US, course of treatment, and the control group. The overall risk of bias from the included studies was high, and the evidence proving these effect calculations were assessed as low quality.

Conclusion: Owing to the high risk of bias and the across-trial heterogeneity of the studies, the current evidence is not clear enough to support US as an effective method to treat MPS. Clinical trials with methodological rigorousness and adequate power are needed to confirm it in the future.

Keywords: ultrasound, myofascial pain syndrome, meta-analysis

\section{Introduction}

Myofascial pain syndrome (MPS) is a local syndrome featured with pain, muscle spasm, oversensitivity, and restricted range of motion due to trigger points (MTrps) on constricted fibers of muscles and fasciae. ${ }^{1,2}$ MPS is the most common cause of back pain, shoulder pain, tension-type headache, and regional pains (e.g., facial pain). ${ }^{2}$ It is one major cause of musculoskeletal system disability as it occurs in $37 \%$ of males and $65 \%$ of females at age $30-60$ years. ${ }^{3}$

Owing to stiff trapezius muscles in most cases, the most common complaint in MPS patients is neck and upper back pains. Although mechanical, nociceptive, and genetic pathologies and primary muscle dysfunctions play a role in the pathogenesis of MPS, the exact mechanisms are still unknown. ${ }^{4,5}$ Therefore, MPS is refractory and the outcomes may 
not always be satisfactory. The therapeutic goals are pain elimination, trigger point deactivation, and release of tight muscle bands. The common methods for the management of MPS are nonsteroidal anti-inflammatory drugs, exercise, acupuncture, trigger point injection, heat packs, and electrotherapy.,7

Ultrasound (US) has been widely popularized and recognized as a non-invasive treatment in the clinical and physiotherapy fields. US is composed of piezoelectric crystals that use high-frequency alternative current to transform electrical energy to mechanical oscillation energy. ${ }^{8}$ The thermal and non-thermal effects of US would transiently increase the flexibility of tendons, ligaments, and joint capsules, which consequently decreases joint stiffness, pains, and accompanying muscle spasm and temporarily increases blood flow. ${ }^{9}$ The evidences on the effects of US on MPS are still controversial. Some studies demonstrate that the use of US for MPS considerably relieves pain intensity in the upper trapezius muscles (uTMs), ${ }^{10,11}$ but other studies do not show any obvious effect on pains or superiority over placebos. ${ }^{12,13}$

Because of the existence of conflicting evidences and as no available reviews or meta-analyses were done before, it is necessary to reevaluate the existing evidences. Regarding these studies, we comprehensively searched the literature and evaluated the effectiveness of US on pain relief and physical improvement in MPS patients.

\section{Methods}

This meta-analysis was completed in accordance with the Preferred Reporting Items for Systematic Reviews and MetaAnalyses (PRISMA) guidelines. ${ }^{14}$

\section{Literature search}

Two authors (PX and XW) searched through PubMed, Embase and Cochrane Library using keywords and text words related to US, MPS, relevant interventions, and randomized controlled trial (RCT; Supplementary material). The date of the electronic search was from January 1966 to May 2016. The references of relevant studies and reviews were also searched.

\section{Inclusion criteria}

First, two authors (PX and XW) independently checked the abstract and full text of each retrieved article. Any divergence was settled through discussion or consultation with another author (XL). The inclusion criteria were as follows: 1) study design RCT; 2) inclusion of MPS patients; 3) US therapy; 4) report of pain and range of motion (ROM) outcomes; and 5) follow-up for 0 or 12 weeks. Any trial with unbalanced extra modality (e.g., education or exercise) between patients and controls was excluded.

\section{Data collection and analysis}

Two authors (QL and $\mathrm{KC}$ ) independently extracted data from the published reports using a standard data extraction form from Cochrane Collaboration. ${ }^{15}$ They reviewed the study population, types of intervention, outcome measurements, and follow-up. Disagreements were resolved by discussion. Any further information required from the original author was requested by email.

\section{Risk of bias and quality assessment}

Two authors (QL and XL) assessed the methodological quality of each included study. The risk of bias was evaluated from the perspective of randomization rigor, therapy allocation concealment, blinding, data completeness, and data reporting as recommended by Cochrane Collaboration. ${ }^{16}$

Based on the objectives of this review, we established the risk of bias from the key domains of randomization, data completeness, blinding, and therapy allocation concealment. The risk of bias for each article was rated as low (all key domains were adequate), unclear (at least one domain was unclear), and high (at least one domain was inadequate). The risk of bias across articles was rated as low (all articles had low risk), unclear $(>75 \%$ of articles had unclear or low risk and $<25 \%$ of articles had high risk), or high ( $>25 \%$ of articles had high risk). ${ }^{16}$

\section{Outcome measures}

We primarily aimed to investigate the effectiveness of US in pain relief and ROM improvement. The common valid reliable scales for pain intensity assessment and monitoring included numerical rating scale (NRS) and visual analog scale (VAS). They were the primary outcomes in the pain assessment. Furthermore, pressure pain threshold (PPT) was used in some included studies. Therefore, we also used PPT results for the secondary outcome of pain.

In this meta-analysis, the outcome measure for ROM is lateral flexion, rotation, flexion, or extension of cervical joint. If outcomes were reported at several postintervention time nodes in a study, we selected the first and the last measure time nodes (week 0 and week 12). The relative therapeutic effect was measured by the change in pain and ROM scores at the follow-up time node.

\section{Statistical analyses}

The effect estimates were pooled by examining the mean change from baseline to end point of each group. When no standard deviation (SD) of change was available, the SD of baseline measure was used for the meta-analysis and the data were analyzed on RevMan 5.3. ${ }^{17}$ The sufficiently homogeneous data (clinical and statistical) were summarized. 
Continuous data were presented as the standardized mean difference (SMD) with 95\% confidence interval (CI) based on the similarity of the pain and physical function assessment scales. The between-study heterogeneity was measured through $\chi^{2}\left(P<0.10\right.$ : heterogeneity) and $I^{2}$ statistic $\left(I^{2}<40 \%\right.$ : low, $I^{2} \geq 75 \%$ : high). ${ }^{18}$ In case of high heterogeneity, the sources were explored through sensitivity analysis and subgroup analysis. The outcomes were pooled on random-effects models. The significance level was set at $P<0.05$.

\section{Results}

The initial search in May 2016 returned 235 articles (Figure 1). After result combination, removal of duplicates, and review of title and abstract, we included 11 full-text articles. One article without reporting outcomes of pain or function was excluded. ${ }^{8}$ Finally, 10 studies were included, ${ }^{11-13,19-25}$ which were published between 1997 and 2015. The 10 studies were fully analyzed, approved by both reviewers, and the data were extracted. In the end, eight studies ${ }^{11,13,19-23,25}$ were enrolled in quantitative synthesis because the other two studies ${ }^{12,24} \mathrm{did}$ not provide data of effect size.

\section{Description of included studies}

The 10 included RCTs ${ }^{11-13,19-25}$ involving a total of 428 MPS patients are summarized in Table 1 . The mean age of patients among studies was from 21 to 49 years. Nine studies compared US with sham US, and one study compared US with blank control. Four studies ${ }^{11,12,21,24}$ compared US with placebo,

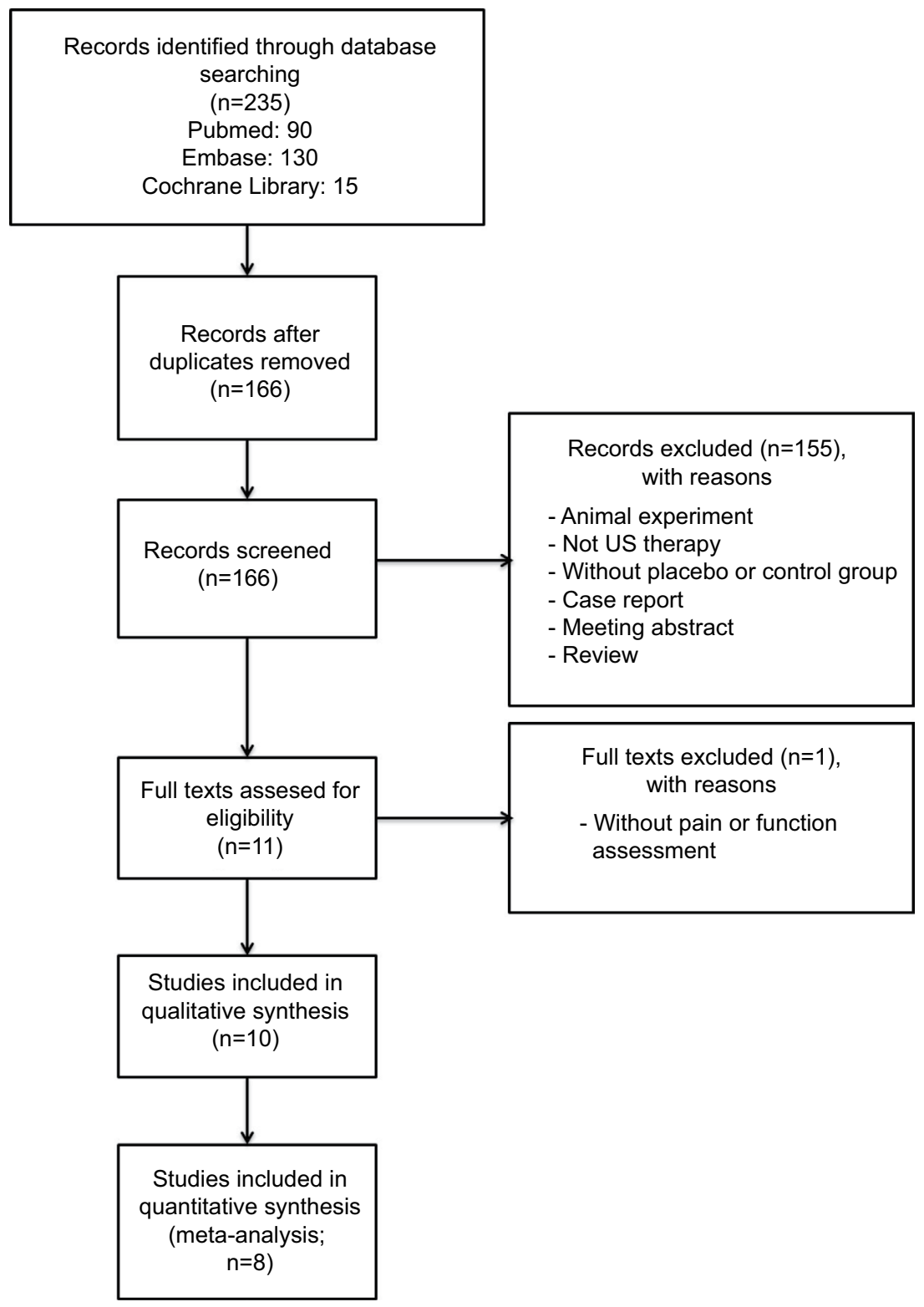

Figure I Flowchart of study selection 
which included co-intervention such as standard stretching and ROM exercises.

Table 1 summarizes the US therapy characteristics of the included studies. The parameter of US was used with pulsed mode (four studies), ${ }^{12,20,22,24}$ continuous mode (four studies), ${ }^{11,23-25}$ and unclear mode (three studies). ${ }^{13,19,21}$ The course of the treatment was once in three studies ${ }^{13,19,20}$ and six to 15 sessions in the other seven studies. ${ }^{11,12,21-25}$

\section{Risk of bias and quality assessment}

The risk of bias was low for three studies ${ }^{23-25}$ unclear for four studies, ${ }^{11,19,21,22}$ and high for three studies ${ }^{12,13,20}$ (Figure 2 and Table 1). Thus, the overall risk of bias from the evidence in this meta-analysis is high.
The evidences for pain and ROM are rated as low quality due to the high risk of bias and the across-trial heterogeneity $\left(P<0.1, I^{2}=65-96 \%\right.$; Figures 3A, 4A, and 5A $)$.

\section{Effect of US on pain}

The effect of US on pain intensity was evaluated by VAS or NRS in eight studies. ${ }^{11-13,21-25}$ The method and total scores of NRS were similar to VAS. Only six studies ${ }^{11,13,21-23,25}$ were included in the meta-analysis because two studies did not report data of effect size. ${ }^{12,24}$ Overall, the application of US has significant impact on pain relief at rest or activity condition (SMD [CI] $=-1.41[-2.15,0.67], P=0.0002)$ with a high heterogeneity $\left(\chi^{2}=62.70, P<0.00001, I^{2}=89 \%\right.$; Figure $\left.3 \mathrm{~A}\right)$. Moreover, a subgroup analysis based on follow-up time

Table I Characteristics of included studies

\begin{tabular}{|c|c|c|c|c|c|c|c|c|c|c|}
\hline Reference & Groups & Balance* & $\mathbf{n}^{\dagger}$ & $\begin{array}{l}\text { Age } \\
\text { (years) }\end{array}$ & $\begin{array}{l}\text { Sex } \\
(M / F)\end{array}$ & $\begin{array}{l}\text { BMI } \\
\left(\mathrm{kg} / \mathrm{m}^{2}\right)\end{array}$ & $\begin{array}{l}\text { Parameters of } \\
\text { intervention }\end{array}$ & Outcomes & Test time ${ }^{\#}$ & $\begin{array}{l}\text { Risk of } \\
\text { bias }\end{array}$ \\
\hline $\begin{array}{l}\text { Kavadar et al } \\
(2015)^{25}\end{array}$ & $\begin{array}{l}\text { GI: CUS } \\
\text { G2: sham }\end{array}$ & NR & $\begin{array}{l}30 \\
29\end{array}$ & $\begin{array}{l}37.43 \\
35.83\end{array}$ & $\begin{array}{l}6 / 24 \\
4 / 25\end{array}$ & $\begin{array}{l}23.9 \\
25.2\end{array}$ & $\begin{array}{l}\mathrm{I} .5 \mathrm{~W} / \mathrm{cm}^{2} \text {, } \\
\mathrm{I} \mathrm{MHz}, 6 \mathrm{~min}, \mathrm{I} 5 \\
\text { sessions }\end{array}$ & $\begin{array}{l}\text { VAS (rest, activity), } \\
\text { PPT, 0-5 scale, BDS }\end{array}$ & 0,3 months & Low \\
\hline $\begin{array}{l}\text { llter et al } \\
(2015)^{24}\end{array}$ & $\begin{array}{l}\text { GI: CUS } \\
\text { G2: PUS } \\
\text { G3: sham }\end{array}$ & Exercise & $\begin{array}{l}20 \\
20 \\
20\end{array}$ & $\begin{array}{l}33 \\
32 \\
33\end{array}$ & $\begin{array}{l}8 / 12 \\
4 / 16 \\
6 / 14\end{array}$ & NR & $\begin{array}{l}\text { I } \mathrm{W} / \mathrm{cm}^{2}, 3 \mathrm{MHz} \text {, } \\
5 \mathrm{~min}, 10 \text { sessions } \\
\text { (within } 2 \text { weeks) }\end{array}$ & $\begin{array}{l}\text { VAS (rest, activity), } \\
\text { five-step scale, BDS, } \\
\text { NHP, NPDS }\end{array}$ & $\begin{array}{l}0,6 \\
12 \text { weeks }\end{array}$ & Low \\
\hline $\begin{array}{l}\text { Manca et al } \\
(2014)^{23}\end{array}$ & $\begin{array}{l}\text { GI: CUS } \\
\text { G2: sham }\end{array}$ & NR & $\begin{array}{l}12 \\
12\end{array}$ & $\begin{array}{l}24.5 \\
26\end{array}$ & $\begin{array}{l}5 / 7 \\
6 / 6\end{array}$ & NR & $\begin{array}{l}\mathrm{I} .5 \mathrm{~W} / \mathrm{cm}^{2}, \\
3 \mathrm{MHz}, 12 \mathrm{~min}, \\
10 \text { sessions } \\
\text { (within } 2 \text { weeks) }\end{array}$ & $\begin{array}{l}\text { NRS, PPT, cervical } \\
\text { joint ROM }\end{array}$ & 0,12 weeks & Low \\
\hline $\begin{array}{l}\text { Sarrafzadeh } \\
\text { et al }(2012)^{22}\end{array}$ & $\begin{array}{l}\text { GI: PUS } \\
\text { G2: blank }\end{array}$ & NR & $\begin{array}{l}15 \\
15\end{array}$ & $\begin{array}{l}21.47 \\
22.2\end{array}$ & $\begin{array}{l}\text { Only } \\
\text { females }\end{array}$ & $\begin{array}{l}20.5 \\
20.4\end{array}$ & $\begin{array}{l}\text { I. } 2 \mathrm{~W} / \mathrm{cm}^{2} \text {, } \\
\text { I } \mathrm{MHz}, 5 \mathrm{~min}, \text { six } \\
\text { sessions }\end{array}$ & $\begin{array}{l}\text { VAS, PPT, cervical } \\
\text { joint ROM }\end{array}$ & 0 week & Unclear \\
\hline $\begin{array}{l}\text { Ay et al } \\
(20 I I)^{2 I}\end{array}$ & $\begin{array}{l}\text { GI: US } \\
\text { (unclear } \\
\text { mode) } \\
\text { G2: sham }\end{array}$ & Exercise & 20 & 49.45 & $4 / 16$ & NR & $\begin{array}{l}1.5 \mathrm{~W} / \mathrm{cm}^{2} \text {, } \\
\text { I } \mathrm{MHz}, 10 \mathrm{~min}, \\
15 \text { sessions } \\
\text { (within } 3 \text { weeks) }\end{array}$ & $\begin{array}{l}\text { VAS, PPT, NPDS, } \\
\text { cervical joint ROM, } \\
\text { numbers of trigger } \\
\text { points }\end{array}$ & 0 week & Unclear \\
\hline $\begin{array}{l}\text { Dündar et al } \\
(2010)^{11}\end{array}$ & $\begin{array}{l}\text { GI: CUS } \\
\text { G2: sham }\end{array}$ & Exercise & $\begin{array}{l}28 \\
27\end{array}$ & $\begin{array}{l}36.6 \\
35.8\end{array}$ & $\begin{array}{l}8 / 20 \\
8 / 19\end{array}$ & NR & $\begin{array}{l}\mathrm{I} .5 \mathrm{~W} / \mathrm{cm}^{2} \text {, } \\
\mathrm{I} \mathrm{MHz}, 8 \mathrm{~min}, \mathrm{I} 5 \\
\text { sessions (within } \\
3 \text { weeks) }\end{array}$ & $\begin{array}{l}\text { VAS (rest, activity), } \\
\text { cervical joint ROM, } \\
\text { NDI, NHP }\end{array}$ & 0,12 weeks & Unclear \\
\hline $\begin{array}{l}\text { Aguilera et al } \\
(2009)^{20}\end{array}$ & $\begin{array}{l}\text { GI: PUS } \\
\text { G2: sham }\end{array}$ & NR & $\begin{array}{l}22 \\
22\end{array}$ & $\begin{array}{l}39 \\
34.7\end{array}$ & $\begin{array}{l}10 / 12 \\
10 / 12\end{array}$ & NR & $\begin{array}{l}\mathrm{I} \mathrm{W} / \mathrm{cm}^{2}, \mathrm{I} \mathrm{MHz}, \\
2 \mathrm{~min}\end{array}$ & $\begin{array}{l}\text { PPT, cervical joint } \\
\text { ROM, BEA }\end{array}$ & 0 week & High \\
\hline $\begin{array}{l}\text { Srbely et al } \\
(2008)^{19}\end{array}$ & $\begin{array}{l}\text { Gl: US } \\
\text { (unclear } \\
\text { mode) } \\
\text { G2: sham }\end{array}$ & NR & 25 & 42.9 & $13 / 12$ & 23.5 & $\begin{array}{l}0.52 \mathrm{~W} / \mathrm{cm}^{2}, \\
\mathrm{I} \mathrm{MHz}, 10 \mathrm{~min}\end{array}$ & PPT & $\begin{array}{l}1,3,5,10 \\
15 \min \end{array}$ & Unclear \\
\hline $\begin{array}{l}\text { Gam et al } \\
(1998)^{12}\end{array}$ & $\begin{array}{l}\text { GI: PUS } \\
\text { G2: sham }\end{array}$ & Exercise & $\begin{array}{l}18 \\
22\end{array}$ & $\begin{array}{l}39.5 \\
42\end{array}$ & NR & NR & $\begin{array}{l}3 \mathrm{~W} / \mathrm{cm}^{2}, 100 \mathrm{~Hz} \text {, } \\
15 \mathrm{~min} \text {, eight } \\
\text { sessions (within } \\
4 \text { weeks) }\end{array}$ & $\begin{array}{l}\text { VAS (rest, activity), } \\
\text { numbers of trigger } \\
\text { points }\end{array}$ & 0,6 weeks & High \\
\hline $\begin{array}{l}\text { Lee et al } \\
(1997)^{13}\end{array}$ & $\begin{array}{l}\text { GI: US } \\
\text { (unclear } \\
\text { mode) } \\
\text { G2: sham }\end{array}$ & NR & $26^{\$}$ & $43.7^{\$}$ & $12 / 14^{\$}$ & NR & $0.5 \mathrm{~W} / \mathrm{cm}^{2}, 6 \mathrm{~min}$ & $\begin{array}{l}\text { VAS, PPT, cervical } \\
\text { joint ROM }\end{array}$ & 0 week & High \\
\hline
\end{tabular}

Notes: *All patients accepted usual care, which was balanced between groups. ${ }^{*} 0$ means at the end of the treatment. ${ }^{\$}$ Only data from the whole trial are available. ${ }^{t} n=$ number of patients. Age and BMI data are shown as mean.

Abbreviations: BDS, Beck's depression scale; BEA, basal electrical activity; BMI, body mass index; CUS, continuous ultrasound; F, female; G, group; M, male; NDI, neck disability index; NHP, Nottingham health profile; NPDS, neck pain and disability scale; NR, not reported; NRS, numerical rating scale; PPT, pressure pain threshold; PUS, pulsed ultrasound; ROM, range of motion; US, ultrasound; VAS, visual analog scale. 
A

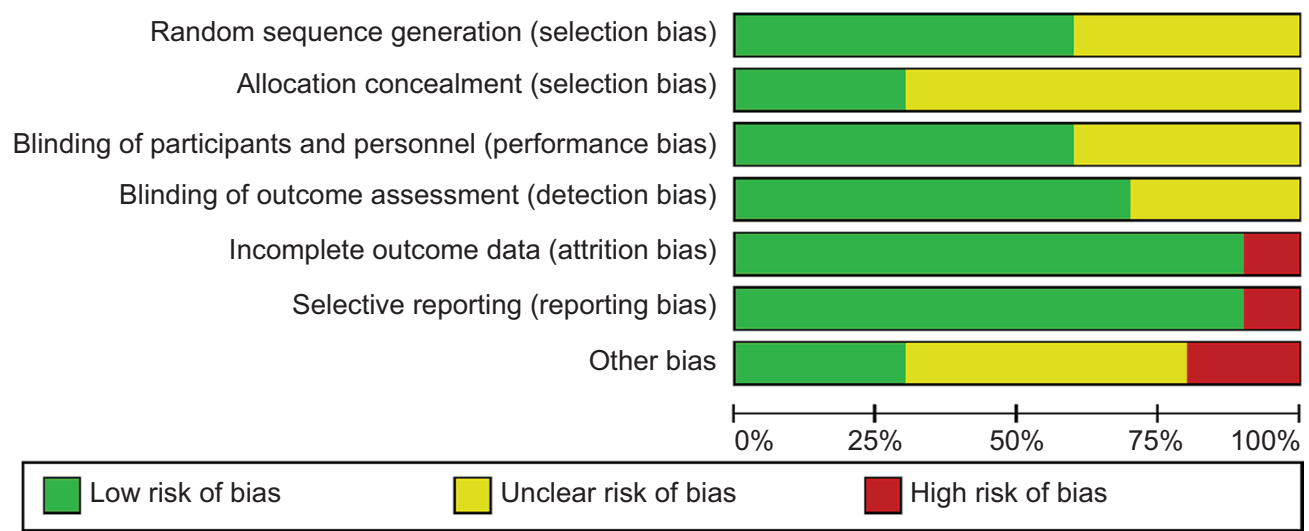

B

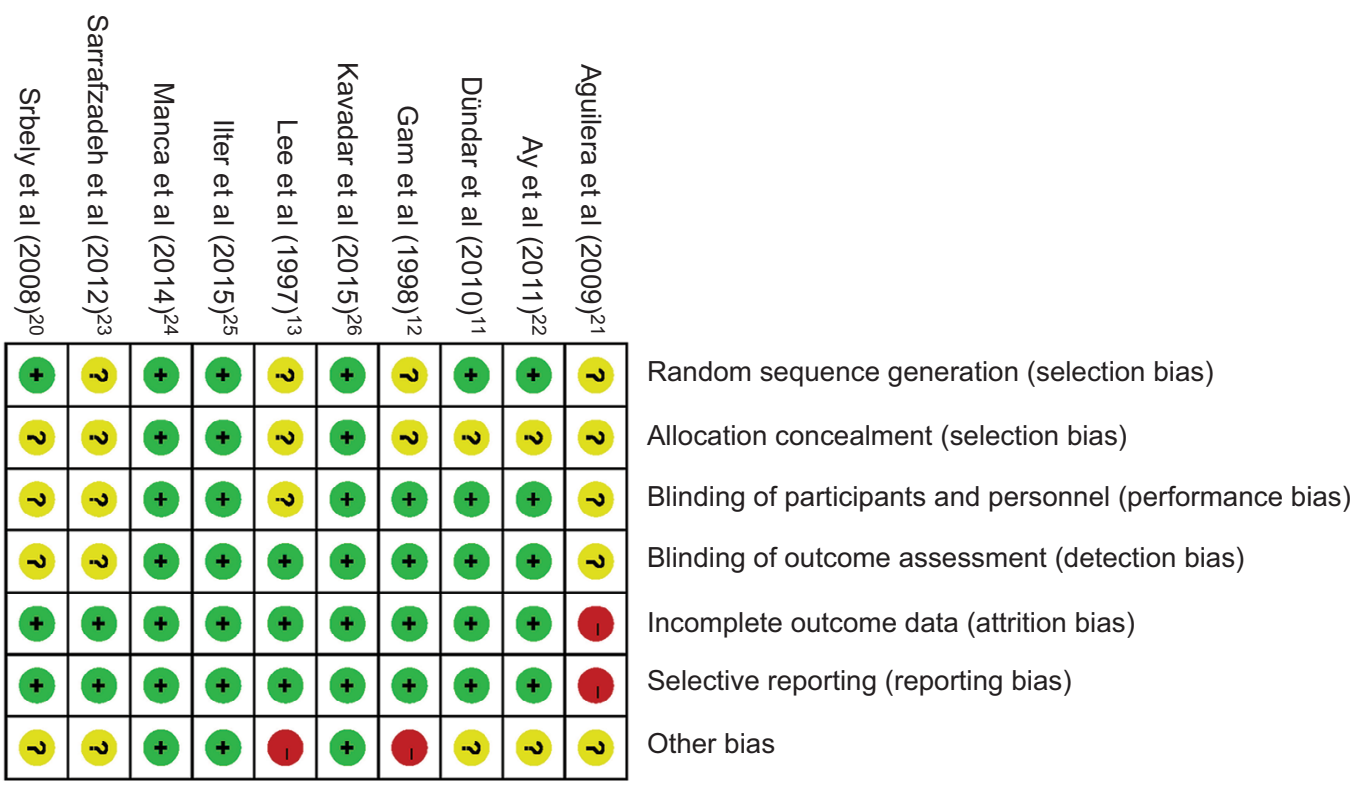

Low risk of bias

? Unclear risk of bias

High risk of bias

Figure 2 Risk of bias assessment.

Notes: (A) Risk of bias graph: review authors' judgments about each risk of bias item presented as percentages across all included studies. (B) Risk of bias graph: review authors' judgments about each risk of bias item for each included study.

showed a significant difference in favor of US versus non-US at week 0 (assessment immediately after treatment), SMD $(\mathrm{CI})=-0.85(-1.52,-0.18), P=0.01$, and week 12 , SMD $(\mathrm{CI})=-1.48(-2.92,-0.03), P=0.04$, with significant heterogeneity $\left(\chi^{2}=27.97, P<0.0001, P=82 \% ; \chi^{2}=24.95, P<0.00001\right.$, $I^{2}=92 \%$; Figure $\left.3 \mathrm{~B}\right)$. The high heterogeneity was then assessed via sensitivity analysis. After excluding two studies ${ }^{11,13}$, the use of US still resulted in a significant decrease in pain intensity (SMD $[\mathrm{CI}]=-1.96[-2.50,-1.43], P<0.00001)$ with no heterogeneity $\left(\chi^{2}=5.12, P=0.16, P^{2}=41 \%\right.$; Figure $\left.3 \mathrm{C}\right)$.

PPT was used for pain assessment in seven studies. ${ }^{13,19-23,25}$ All the seven studies were included in the meta-analysis, and a subgroup analysis based on follow-up time was done. Results showed that US versus non-US significantly increased pain threshold at week 0 (SMD $[\mathrm{CI}]=1.08[0.55$, $1.60], P<0.0001)$ with a high heterogeneity $\left(\chi^{2}=23.59\right.$,
$P=0.0006, I^{2}=75 \%$; Figure 4A). However, no significant effect of US on pain threshold was found at week 12 (SMD $[\mathrm{CI}]=0.88[-0.80,2.56], P=0.31)$ with a high heterogeneity $\left(\chi^{2}=11.29, P=0.0008, I^{2}=91 \%\right.$; Figure $\left.4 \mathrm{~A}\right)$. The sensitivity analysis showed that after the exclusion of two studies, ${ }^{22,25}$ the application of US significantly increased pain threshold (SMD [CI] $=0.71[0.41,1.00], P<0.00001)$ at week 0 with no heterogeneity $\left(\chi^{2}=2.19, P=0.70, I^{2}=0 \%\right.$; Figure 4B).

\section{Effect of US on ROM}

The effect of US on cervical joint ROM was assessed in six studies, ${ }^{11,13,20-23}$ and the date of ROM change from baseline was extracted. Figure 5 shows the outcomes of meta-analysis for cervical joint ROM. The subgroup analysis showed that US or US+exercise versus non-US had no significant effect on lateral flexion $(\mathrm{SMD}[\mathrm{CI}]=0.40[-0.19,0.99], P=0.19)$, rotation (SMD 


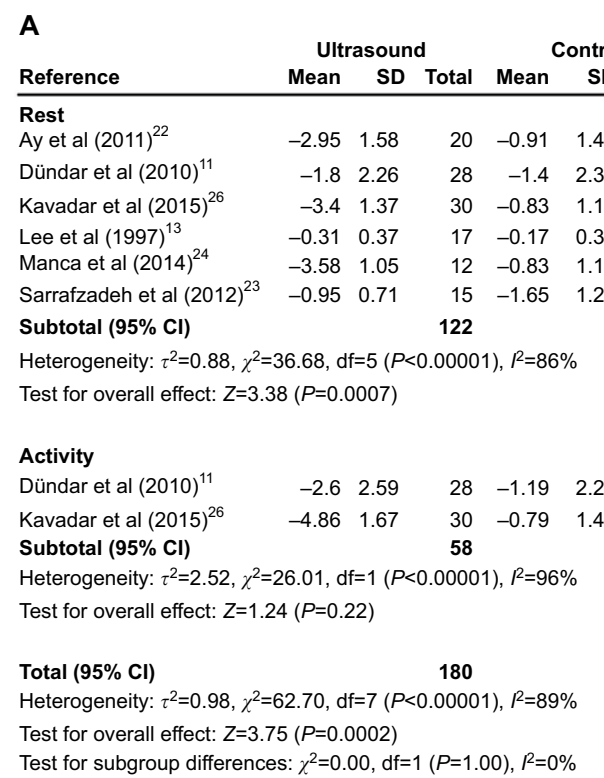

$170 \quad 100.0 \%$

SMD

IV, random, $95 \% \mathrm{CI}$

SMD

IV, random, $95 \% \mathrm{CI}$

Test for subgroup differences: $\chi^{2}=0.00, \mathrm{df}=1(P=1.00), P^{2}=0 \%$

$\begin{array}{rrr}20 & 12.8 \% & -1.32[-2.01,-0.63] \\ 27 & 13.4 \% & -0.17[-0.70,-0.36] \\ 29 & 13.0 \% & -2.00[-2.63,-1.36] \\ 11 & 12.4 \% & -0.36[-1.12,-0.41] \\ 12 & 10.9 \% & -2.40[-3.49,-1.31] \\ 15 & 11.4 \% & -2.52[-3.51,-1.53] \\ \mathbf{1 1 4} & \mathbf{7 3 . 9 \%} & \mathbf{- 1 . 4 1 [ - 2 . 2 3 , - 0 . 5 9 ]}\end{array}$

\section{B}

Ultrasound Control

Reference

Mean SD Total Mean SD Total Weight

0 weeks

Ay et al $(2011)^{22}$

Dündar et al $(2010)^{11}$

Kavadar et al $(2015)^{26}$

Lee et al (1997) ${ }^{13}$

Manca et al (2014)

$\begin{array}{lllllll}-2.95 & 1.58 & 20 & -0.91 & 1.45 & 20 & 11.4 \%\end{array}$

SMD

Sarrafzadeh et al $(2012)^{23}$

$\begin{array}{lll}28 & -1.2 & 2.3\end{array}$

$27 \quad 12.1 \%$

$\begin{array}{lllllll}-3.9 & 1.48 & 30 & -2.27 & 1.29 & 29 & 12.0 \%\end{array}$

$\begin{array}{lllllll}-0.31 & 0.37 & 17 & -0.17 & 0.39 & 11 & 11.0 \%\end{array}$

$\begin{array}{lllllll}-2.01 & 1.07 & 12 & -2.27 & 1.29 & 12 & 10.8 \%\end{array}$

Subtotal $(95 \% \mathrm{Cl})$

$\begin{array}{lll}15 & 1.65 & 1.23\end{array}$

122

$\begin{array}{rr}15 & 9.8 \% \\ 114 & 67.0 \%\end{array}$

$-1.32[-2.01,-0.63]$

$-0.18[-0.71,0.35]$

$-0.16[-1.71,-0.60]$

$-0.36[-1.12,0.41]$

$0.21[-0.59,1.01]$

$-2.52[-3.51,-1.53]$

Heterogeneity: $\tau^{2}=0.56, \chi^{2}=27.97, \mathrm{df}=5(P<0.0001), P=82 \%$

$-0.85[-1.52,-0.18]$

Test for overall effect: $Z=2.49(P=0.01)$

12 weeks

Dündar et al (2010)

Kavadar et al $(2015)^{26}$

Manca et al (2014) ${ }^{24}$

Subtotal $(95 \% \mathrm{Cl})$

$\begin{array}{lllll}-1.8 & 2.26 & 28 & -1.4 & 2.33\end{array}$

$\begin{array}{lllll}-3.4 & 1.37 & 30 & -0.83 & 1.16\end{array}$

$\begin{array}{lllll}-3.58 & 1.05 & 12 & -0.83 & 1.16\end{array}$

70

$27 \quad 12.1 \%$

$29 \quad 11.6 \%$

$12 \quad 9.3 \%$

$68 \quad 33.0 \%$

$-2.17[-0.70,-0.36]$

$-2.00[-2.63,-1.36]$

$-2.40[-3.49,-1.31]$

$-1.48[-2.92,-0.03]$

Heterogeneity: $\tau^{2}=1.47, \gamma^{2}=24.95, \mathrm{df}=2(P<0.00001), l^{2}=92 \%$

Test for overall effect: $Z=2.01(P=0.04)$

Total $(95 \% \mathrm{Cl})$

$182 \quad 100.0 \%$

$-1.05[-1.65,-0.45]$

Heterogeneity: $\tau^{2}=0.70, \chi^{2}=54.98, \mathrm{df}=8(P<0.00001), I^{2}=85 \%$

Test for overall effect: $Z=3.42(P=0.0006)$

Test for for subgroup differences: $\chi^{2}=0.60, \mathrm{df}=1(P=0.044), P=0 \%$

C

\begin{tabular}{lll} 
Ultrasound & \multicolumn{2}{c}{ Control } \\
Reference & Mean SD Total Mean SD
\end{tabular}

Total Weight

SMD

\begin{tabular}{llllllll}
\hline Ay et al $(2011)^{22}$ & -2.95 & 1.58 & 20 & -0.91 & 1.45 & 20 & $30.2 \%$
\end{tabular}

$\begin{array}{llllllll}\text { Kavadar et al }(2015)^{26} & -3.4 & 1.37 & 30 & -0.83 & 1.16 & 29 & 32.9 \%\end{array}$

$\begin{array}{llllllll}\text { Manca et al }(2014)^{24} & -3.58 & 1.05 & 12 & -0.83 & 1.16 & 12 & 17.2 \%\end{array}$

$\begin{array}{llllllll}\text { Sarrafzadeh et al }(2012)^{23} & -0.95 & 0.71 & 15 & -1.65 & 1.23 & 15 & 19.7 \%\end{array}$

Total $(95 \% \mathrm{Cl})$

77

$76 \quad 100.0 \%$

IV, random, $95 \% \mathrm{Cl}$

dom, $95 \% \mathrm{Cl}$

Heterogeneity: $\tau^{2}=0.12, \chi^{2}=5.12, \mathrm{df}=3(P=0.16), P^{2}=41 \%$

Test for overall effect: $Z=7.21(P<0.00001)$

$-1.32[-2.01,-0.63]$

$-2.00[-2.63,-1.36]$

$-2.40[-3.49,-1.31]$

$-2.52[-3.51,-1.53]$

$-1.96[-2.50,-1.43]$

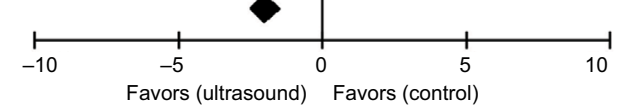

Figure 3 Meta-analyses of US therapy on pain intensity (VAS or NRS).

Notes: (A) SMDs of at rest and activity. (B) SMDs at 0 and 12 weeks. (C) SMDs at rest after excluding two studies.

Abbreviations: $\mathrm{Cl}$, confidence interval; IV, inverse variance; NRS, numerical rating scale; SD, standard deviation; SMD, standardized mean difference; US, ultrasound; VAS, visual analog scale. 


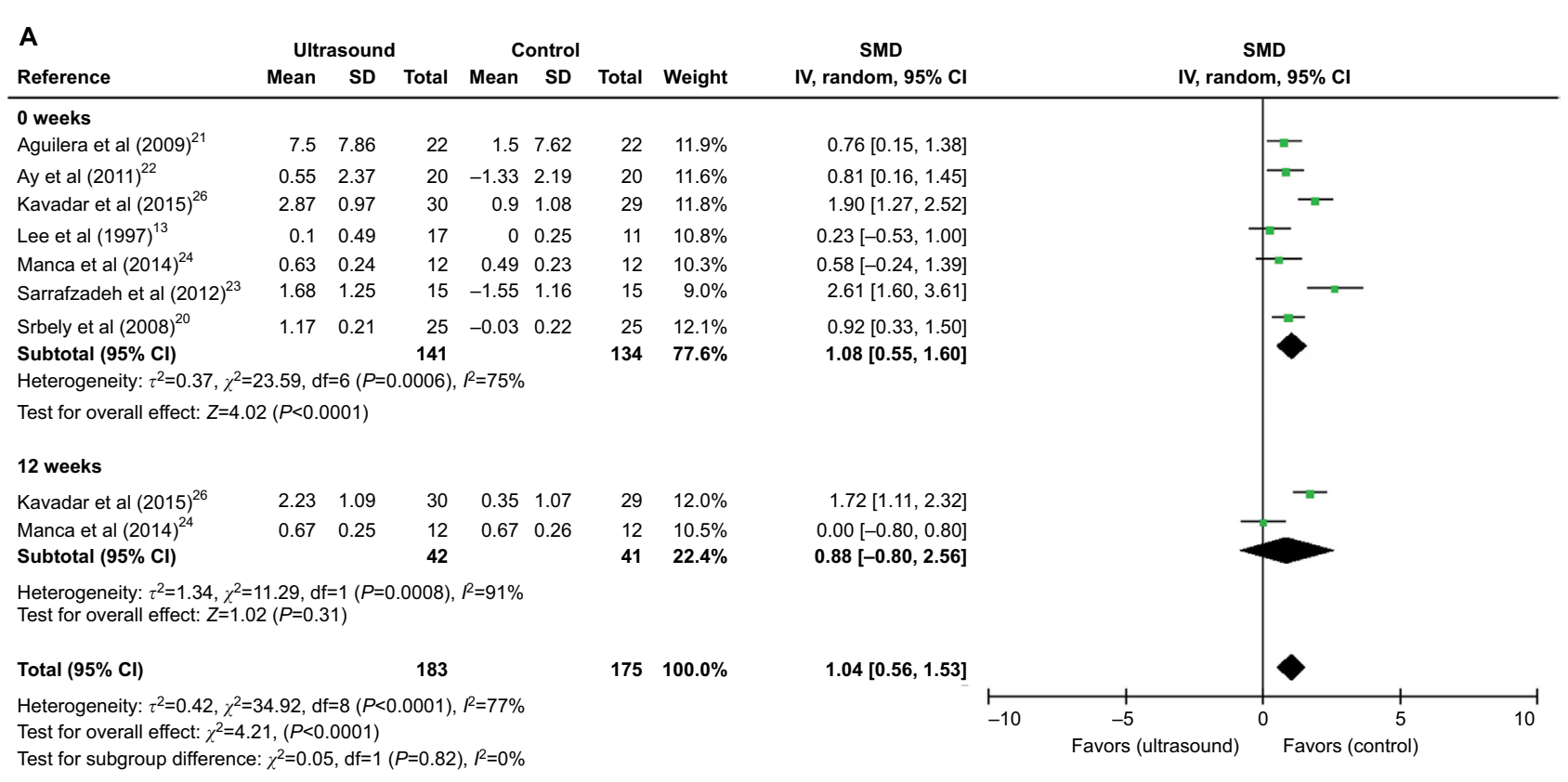

\begin{tabular}{|c|c|c|c|c|c|c|c|}
\hline \multirow[b]{2}{*}{ Reference } & \multicolumn{3}{|c|}{ Ultrasound } & \multicolumn{3}{|c|}{ Control } & \multirow[b]{2}{*}{ Weigh } \\
\hline & Mean & SD & Total & Mean & SD & Total & \\
\hline Aguilera et al $(2009)^{21}$ & 7.5 & 7.86 & 22 & 1.5 & 7.62 & 22 & $23.7 \%$ \\
\hline Ay et al $(2011)^{22}$ & 0.55 & 2.37 & 20 & -1.33 & 2.19 & 20 & $21.4 \%$ \\
\hline Lee et al $(1997)^{13}$ & 0.1 & 0.49 & 17 & 0 & 0.25 & 11 & $15.4 \%$ \\
\hline Manca et al $(2014)^{24}$ & 0.63 & 0.24 & 12 & 0.49 & 0.23 & 12 & $13.3 \%$ \\
\hline Srbely et al $(2008)^{20}$ & 0.17 & 0.21 & 25 & -0.03 & 0.22 & 25 & $26.1 \%$ \\
\hline Total $(95 \% \mathrm{Cl})$ & & & 96 & & & 90 & $100.0 \%$ \\
\hline \multicolumn{8}{|c|}{ Heterogeneity: $\chi^{2}=2.19, \mathrm{df}=4(P=0.70), P^{2}=0 \%$} \\
\hline \multicolumn{8}{|c|}{ Test for overall effect: $Z=4.62,(P<0.00001)$} \\
\hline
\end{tabular}

Figure 4 Meta-analyses of US therapy on pain threshold (PPT).

Notes: (A) SMDs at 0 and 12 weeks. (B) SMDs at 0 week after excluding two studies.

Abbreviations: $\mathrm{Cl}$, confidence interval; IV, inverse variance; PPT, pressure pain threshold; SD, standard deviation; SMD, standardized mean difference; US, ultrasound.

$[\mathrm{CI}]=0.10[-0.33,0.52], P=0.66)$, or extension or flexion (SMD $[\mathrm{CI}]=0.16[-0.35,0.68], P=0.53)$ with or without heterogeneity present $\left(\chi^{2}=22.32, P=0.00005, P=78 \% ; \chi^{2}=1.10, P=0.30\right.$, $P^{2}=9 \% ; \chi^{2}=1.58, P=0.21, P^{2}=37 \%$; Figure $\left.5 \mathrm{~A}\right)$. The sensitivity analysis showed no significant difference between US and nonUS at lateral flexion (SMD [CI $]=0.09[-0.20,0.38], P=0.54$ ) with no heterogeneity $\left(\chi^{2}=2.19, P=0.70, I^{2}=0 \%\right)$ after exclusion of one study ${ }^{23}$ (Figure 5B).

\section{Discussion}

To our knowledge, this is the first systematic review comprehensively evaluating the effects of US therapy on pain and ROM of MPS patients. The overall results indicate that US is effective in pain relief, but not in ROM improvement. However, due to the high risk of bias and the across-trial heterogeneity of the studies, the current evidence is not clear enough to support US as an effective method to treat MPS.

In the past years, the treatment of MPS was always a difficult problem. Since the etiology of MPS is unknown, the treatment protocols mostly target at symptoms. The physical treatment modalities are aimed at relax of rigid bands, inactivation of trigger points, and regulation of predisposing factors. ${ }^{26,27}$ Although US is commonly used for physical therapy, two reviews found insufficient evidence that US is more therapeutically effective than placebo on musculoskeletal problems. ${ }^{28,29}$ Some metaanalysis and systematic reviews state that comparison of findings is complicated due to technical differences in the dose of US, treatment duration, the area and type of the treated tissue, and combination of US with other treatment types. ${ }^{28,30}$

In recent years, US has been applied in MPS treatment. Some clinical trials prove the benefits of US for MPS patients, ${ }^{11,19-25}$ but other studies indicate that US has no significant effect on MPS. ${ }^{12,13}$ Furthermore, there is a lack of large-scale RCTs. The effect of US on MPS is not conclusive due to the small number of investigated patients. As we know, the present review involving 10 RCTs aimed to uncover the effect of US on MPS. This review and meta-analysis has some strengths, such as transparent comprehensive search, 


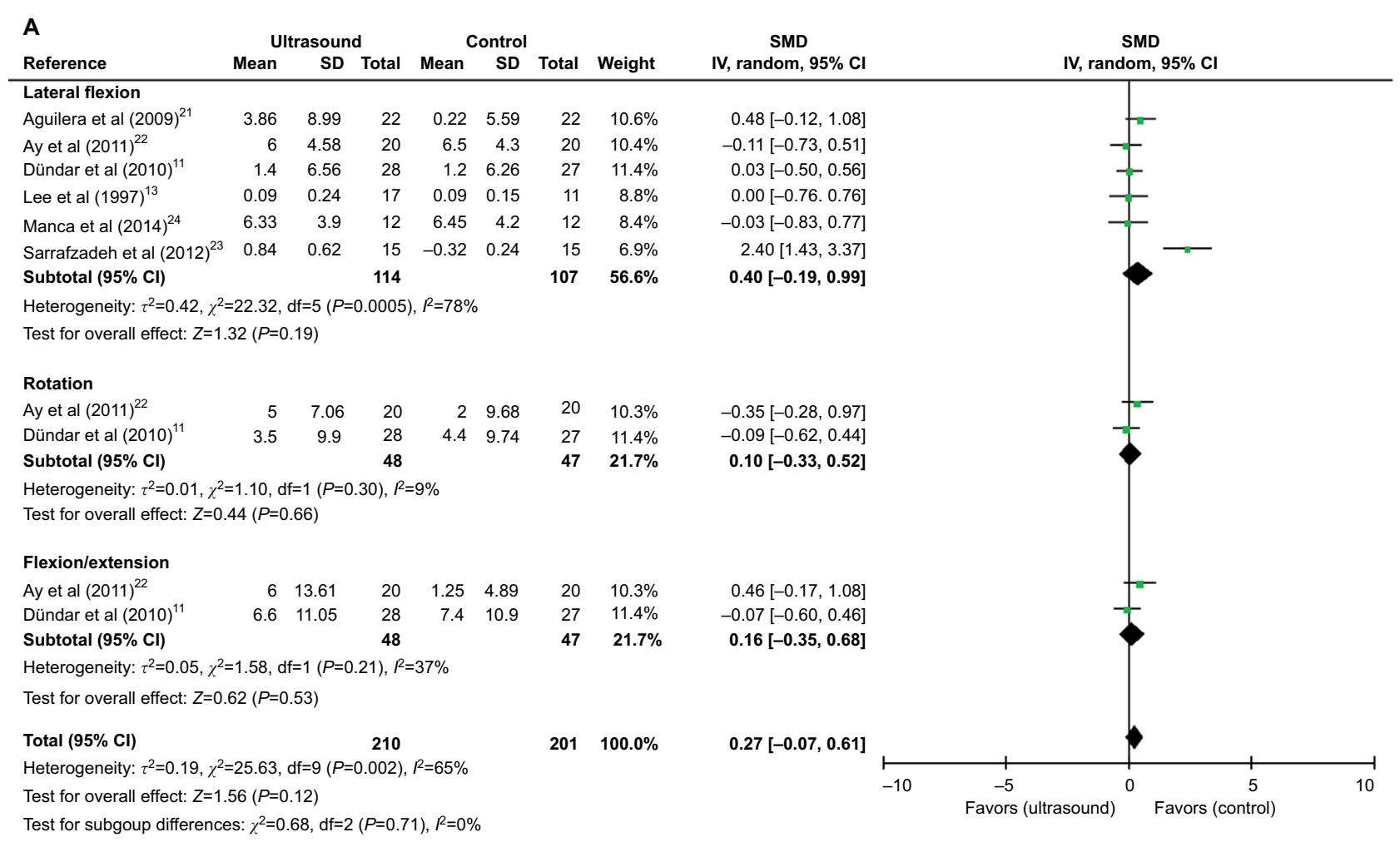

B

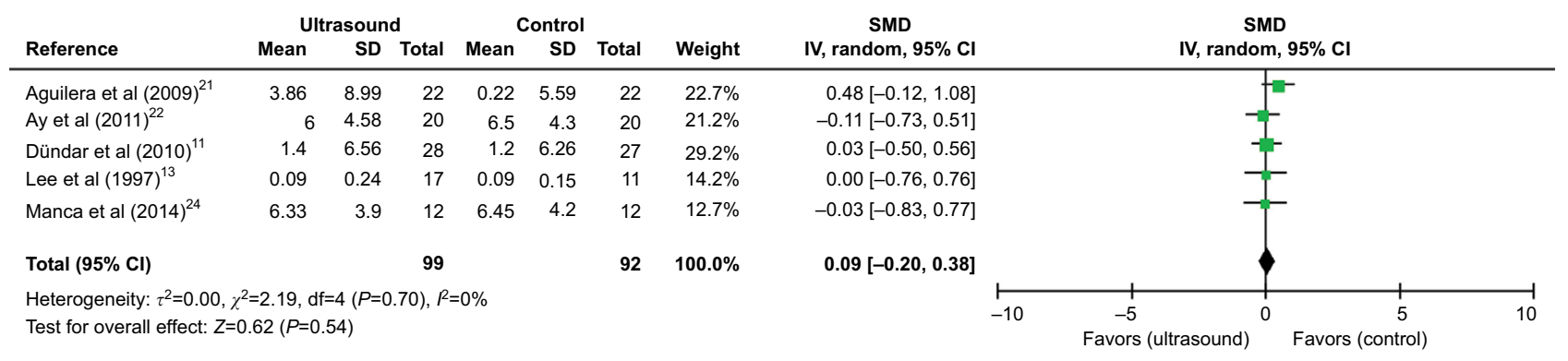

Figure 5 Meta-analyses of US therapy on cervical joint ROM at the last follow-up time.

Notes: (A) SMDs at lateral flexion, rotation, flexion, or extension. (B) SMDs at lateral flexion after excluding one study.

Abbreviations: $\mathrm{Cl}$, confidence interval; IV, inverse variance; ROM, range of motion; SD, standard deviation; SMD, standardized mean difference; US, ultrasound.

independent and duplicate study inclusion and data collection, and the use of standard meta-analytic techniques for effectiveness evaluation. A total of 10 studies exploring the effects of US on MPS were included in this review. The risk of bias was low in three studies, unclear in four studies, and high in three studies.

MPS is typically manifested as deep somatic intensityvarying pains that are tensive, constrictive or cramp-like, or well discriminated, and its onset is sudden or gradual, continuous or intermittent, and at rest or only during movement. ${ }^{31,32}$ Eight studies ${ }^{11-13,21-25}$ used VAS or NRS to evaluate pain intensity, but only six studies were incorporated into the meta-analysis. The estimates of meta-analysis suggested that US has a significant benefit on pain relief of MPS patients at the final follow-up. The subgroup analy- sis showed that US also has a significant benefit on pain relief at the end of treatment and 12 weeks follow-up. The heterogeneity among studies by the $I^{2}$ statistic indicated that the results should be interpreted with caution. After the exclusion of two studies, ${ }^{11,13}$ the pooled results with no heterogeneity from four studies showed that US versus nonUS could relieve the self-reported pain. However, this result was restricted by the low number of included studies. The inconsistency and heterogeneity between these two studies and other studies might be attributed to the differences in the parameter of US, treatment course, and pain duration. Two studies reporting VAS were excluded from the quantitative synthesis due to the lack of data. As reported in these studies, continuous US therapy is more efficient in pain relief than pulsed US or sham therapy, but the latter two treatments are 
not significantly different. ${ }^{24}$ Moreover, pulsed US therapy gives no pain reduction in MPS patients. ${ }^{12}$ The conclusions of these two studies were mainly limited by the type of statistical data or high risk of bias.

The sensory changes at trigger and target levels have been extensively studied by detecting pain thresholds after both muscles and overlying superficial tissues (subcutaneous tissue and skin) were subjected to different stimuli. As extensively reported, the PPT is reduced at the trigger point level. ${ }^{33,34}$ PPT was used for pain assessment in seven included studies, ${ }^{11-13,21-23,25}$ which were all incorporated into metaanalysis. Overall, a significant positive effect of US therapy versus non-US was observed. Moreover, US seemed more efficient on pain threshold elevation at the end of treatment, but had no significant benefit after 12 weeks of follow-up. These results are consistent with the finding about the effect of US on pain assessed by VAS or NRS. However, the interpretation of these results is also limited by the high heterogeneity. As for the source of heterogeneity, no heterogeneity or outcome change was found after exclusion of two studies. ${ }^{11,13}$ The inconsistency and heterogeneity between these two studies and the other studies might be explained by the differences in the sex of participants, method of PPT measurement, parameter of US, treatment course, pain duration, and the control group.

In addition to the pain, MPS patients also suffer from the altered motor function, which is manifested as weakness, work tolerance reduction, and inability to coordinate TrPs-harboring muscles. ${ }^{35}$ All of the patients with regional neck pain in our review presented with $\operatorname{TrPs}$ in the uTM. Therefore, cervical joint ROM is an important outcome of physical function in our review, but it was reported in only six studies, ${ }^{11,13,20-23}$ which were included in the quantitative synthesis. Pooled comparison of the ROM of lateral flexion, rotation, flexion or extension in six studies showed that US therapy was not more effective than the control group. The high heterogeneity in the pooled results of lateral flexion was then explored via sensitivity analysis. After exclusion of one study, the meta-analysis showed no significant difference in the ROM of lateral flexion without heterogeneity. The inconsistency and heterogeneity between this study and the other studies might be attributed to the differences in sex, parameter of US, treatment course, and the control group. However, due to the small sample size, the effect of US versus non-US on physical function is inconclusive.

This review has some limitations. First, the findings are based on evidence, which is of high risk of bias and low quality. The small number of included studies due to high heterogeneity may also limit the findings. This indicates that the effect sizes for pain might be partially aggravated by the method limitations of the included studies. Moreover, we used the cervical joint ROM to compare US therapy with non-US therapy. However, there is conflicting evidence about the prevalence of restricted ROM in MPS. ${ }^{36}$ Furthermore, the other outcome measures besides ROM may be considered so as to assess the effects of US on MPS.

Another limitation is that the findings from the articles comparing US and placebo and the articles including cointervention were pooled. We assumed that there was no interaction between US and exercise, but we could not verify this assumption. It is well known that exercise contributes to pain relief and ROM improvement for MPS patients. ${ }^{37-39}$ Therefore, we cannot rule out a positive interaction between US and exercise.

Finally, the results were limited by the sexes of study patients. One study ${ }^{22}$ only included female patients, and the number of females was more than that of males in four studies. ${ }^{11,21,24,25}$ The effects of US therapy on MPS may be different in females and males. In addition, the publication bias was not determined owing to the small numbers of included trials and 428 participants. Hence, it is possible that only positive trials, but no negative trials, were published. Overall, the methodological limitations make the effect estimates less convincing.

\section{Conclusion}

The current evidence suggests that there may be a significant effect of US therapy on pain in MPS patients, but not on ROM. The high risk of bias makes the effects of US on pain and ROM inconclusive. The relevant evidence is extremely weak, due to the very low quality of some studies, incompleteness of patient-reported outcomes, and very small sample sizes. Nevertheless, the effects of US therapy on MPS should be confirmed by large-sized and high-quality RCTs that have safeguards against bias and assess important outcomes.

\section{Acknowledgment}

We thank the Nanjing Medical Science and Technology Development Program (YKK15085) for financial support.

\section{Disclosure}

The authors report no conflicts of interest in this work.

\section{References}

1. Stecco A, Gesi M, Stecco C, Stern R. Fascial components of the myofascial pain syndrome. Curr Pain Headache Rep. 2013;17(8):352.

2. Jaeger B. Myofascial trigger point pain. Alpha Omegan. 2013;106(1-2): $14-22$. 
3. Srbely JZ, Dickey JP. Randomized controlled study of the antinociceptive effect of ultrasound on trigger point sensitivity: novel applications in myofascial therapy? Clin Rehabil. 2007;21(5):411-417.

4. Fernandez-de-las-Penas C, Dommerholt J. Myofascial trigger points: peripheral or central phenomenon? Curr Rheumatol Rep. 2014; 16(1):395

5. Pace MC, Mazzariello L, Passavanti MB, et al. Neurobiology of pain. J Cell Physiol. 2006;209(1):8-12.

6. Wheeler AH. Myofascial pain disorders: theory to therapy. Drugs. 2004;64(1):45-62.

7. Lugo LH, Garcia HI, Rogers HL, Plata JA. Treatment of myofascial pain syndrome with lidocaine injection and physical therapy, alone or in combination: a single blind, randomized, controlled clinical trial. BMC Musculoskelet Disord. 2016;17:101.

8. Draper DO, Mahaffey C, Kaiser D, Eggett D, Jarmin J. Thermal ultrasound decreases tissue stiffness of trigger points in upper trapezius muscles. Physiother Theory Pract. 2010;26(3):167-172.

9. Unalan H, Majlesi J, Aydin FY, Palamar D. Comparison of high-power pain threshold ultrasound therapy with local injection in the treatment of active myofascial trigger points of the upper trapezius muscle. Arch Phys Med Rehabil. 2011;92(4):657-662.

10. Majlesi J, Unalan H. High-power pain threshold ultrasound technique in the treatment of active myofascial trigger points: a randomized, double-blind, case-control study. Arch Phys Med Rehabil. 2004;85(5): 833-836.

11. Dündar U, Solak O, Şamli F, Kavuncu V. Effectiveness of ultrasound therapy in cervical myofascial pain syndrome: a double blind, placebocontrolled study. Turk J Rheumatol. 2010;25(3):110-115.

12. Gam AN, Warming S, Larsen LH, et al. Treatment of myofascial trigger-points with ultrasound combined with massage and exercise - a randomised controlled trial. Pain. 1998;77(1):73-79.

13. Lee JC, Lin DT, Hong CZ. The effectiveness of simultaneous thermotherapy with ultrasound and electrotherapy with combined AC and DC current on the immediate pain relief of myofascial trigger points. J Musculoskelet Pain. 1997;5(1):81-90.

14. Moher D, Liberati A, Tetzlaff J, Altman DG. Preferred reporting items for systematic reviews and meta-analyses: the PRISMA statement. BMJ. 2009;339:b2535.

15. Higgins J, Green S. The Cochrane Library. Cochrane Handbook for Systematic Reviews of Intervention 4.2.6. 4 Issue. Chichester: John Wiley \& Sons; 2006.

16. Higgins J, Altman D. Chapter 8: assessing risk of bias in included studies. In: Higgins JPT, Green S, editors. Cochrane Handbook for Systematic Reviews of Interventions. London, UK: The Cochrane Collaboration; 2008:187-241.

17. Review Manager (RevMan) [Computer program]. Version 5.0. Copenhagen: The 436 Nordic Cochrane Centre. The Cochrane Collaboration; 2008.

18. Higgins JP, Thompson SG, Deeks JJ, Altman DG. Measuring inconsistency in meta-analyses. BMJ. 2003;327(7414):557-560.

19. Srbely JZ, Dickey JP, Lowerison M, Edwards AM, Nolet PS, Wong LL. Stimulation of myofascial trigger points with ultrasound induces segmental antinociceptive effects: a randomized controlled study. Pain. 2008;139(2):260-266.
20. Aguilera FJ, Martín DP, Masanet RA, Botella AC, Soler LB, Morell FB. Immediate effect of ultrasound and ischemic compression techniques for the treatment of trapezius latent myofascial trigger points in healthy subjects: a randomized controlled study. J Manipulative Physiol Ther. 2009;32(7):515-520.

21. Ay S, Dogan SK, Evcik D, Baser OC. Comparison the efficacy of phonophoresis and ultrasound therapy in myofascial pain syndrome. Rheumatol Int. 2011;31(9):1203-1208.

22. Sarrafzadeh J, Ahmadi A, Yassin M. The effects of pressure release, phonophoresis of hydrocortisone, and ultrasound on upper trapezius latent myofascial trigger point. Arch Phys Med Rehabil. 2012;93(1):72-77.

23. Manca A, Limonta E, Pilurzi G, et al. Ultrasound and laser as stand-alone therapies for myofascial trigger points: a randomized, double-blind, placebo-controlled study. Physiother Res Int. 2014;19(3):166-175.

24. Ilter L, Dilek B, Batmaz I, et al. Efficacy of pulsed and continuous therapeutic ultrasound in myofascial pain syndrome: a randomized controlled study. Am J Phys Med Rehabil. 2015;94(7):547-554.

25. Kavadar G, Caglar N, Ozen S, et al. Efficacy of conventional ultrasound therapy on myofascial pain syndrome: a placebo controlled study. Agri. 2015;27(4):190-196.

26. Desai MJ, Saini V, Saini S. Myofascial pain syndrome: a treatment review. Pain Ther. 2013;2(1):21-36.

27. Majlesi J, Unalan H. Effect of treatment on trigger points. Curr Pain Headache Rep. 2010;14(5):353-360.

28. Robertson VJ, Baker KG. A review of therapeutic ultrasound: effectiveness studies. Phys Ther. 2001;81(7):1339-1350.

29. van der Windt DA, van der Heijden GJ, van den Berg SG, ter Riet G, de Winter AF, Bouter LM. Ultrasound therapy for musculoskeletal disorders: a systematic review. Pain. 1999;81(3):257-271.

30. Gam AN, Johannsen F. Ultrasound therapy in musculoskeletal disorders: a meta-analysis. Pain. 1995;63(1):85-91.

31. Giamberardino MA, Affaitati G, Fabrizio A, Costantini R. Myofascial pain syndromes and their evaluation. Best Pract Res Clin Rheumatol. 2011;25(2):185-198.

32. Han SC, Harrison P. Myofascial pain syndrome and trigger-point management. Reg Anesth. 1997;22(1):89-101.

33. Reeves J, Jaeger B, Graff-Radford A. Reliability of the pressure algometer as a measure of myofascial trigger point sensitivity. Pain. 1986;24(3):313-321.

34. Hong C, Chen Y, Twehous D. Pressure threshold for referred pain by compression on the trigger point and adjacent areas. J Musculoskelet Pain. 1996;4:61-79.

35. Cummings M, Baldry P. Regional myofascial pain: diagnosis and management. Best Pract Res Clin Rheumatol. 2007;21(2):367-387.

36. Wilke J, Niederer D, Fleckenstein J, et al. Range of motion and cervical myofascial pain. J Bodyw Mov Ther. 2016;20(1):52-55.

37. Acar B, Yilmaz OT. Effects of different physiotherapy applications on pain and mobility of connective tissue in patients with myofascial pain syndrome. J Back Musculoskelet Rehabil. 2012;25(4):261-267.

38. Kim M, Lee M, Kim Y, Oh S, Lee D, Yoon B. Myofascial pain syndrome in the elderly and self-exercise: a single-blind, randomized, controlled trial. J Altern Complement Med. 2016;22(3):244-251.

39. Thompson JM. Exercise in muscle pain disorders. PM R. 2012;4(11): 889-893. 


\section{Supplementary material}

\section{Search strategies for PubMed, Embase and the Cochrane Library database}

\author{
PubMed \\ 1. Myofascial Pain Syndromes [Mesh] \\ 2. Myofascial Pain Syndrome [Title/Abstract] \\ 3. Pain Syndrome, Myofascial [Title/Abstract] \\ 4. Pain Syndromes, Myofascial [Title/Abstract] \\ 5. Syndrome, Myofascial Pain [Title/Abstract] \\ 6. Syndromes, Myofascial Pain [Title/Abstract] \\ 7. Myofascial Trigger Point Pain [Title/Abstract] \\ 8. Trigger Point Pain, Myofascial [Title/Abstract] \\ 9.1 or 2 or 3 or 4 or 5 or 6 or 7 or 8 or 9 \\ 10. ultrasound [Title/Abstract] \\ 11.10 and 11

\section{Embase} \\ 1. "myofascial pain"/exp \\ 2. "pain syndrome, myofascial":ab,ti
}

3. "pain syndromes, myofascial":ab,ti

4. "syndrome, myofascial pain":ab,ti

5. "syndromes, myofascial":ab,ti

6. "myofascial trigger point pain":ab,ti

7. "trigger point pain, myofascial":ab,ti

8.1 or 2 or 3 or 4 or 5 or 6 or 7

9. "ultrasound":ab,ti

10.8 and 9

\section{Cochrane Library}

1. MeSH descriptor: [Myofascial Pain Syndromes] explode all trees

2. Myofascial Pain Syndrome:ti,ab,kw or Pain Syndrome, Myofascial:ti,ab,kw or Pain Syndromes, Myofascial:ti,ab,kw or Syndrome, Myofascial Pain:ti,ab,kw or Syndromes, Myofascial Pain:ti,ab,kw (Word variations have been searched)

3. Myofascial Trigger Point Pain:ti,ab,kw or Trigger Point Pain, Myofascial:ti,ab,kw (Word variations have been searched)

4. ultrasound:ti,ab,kw (Word variations have been searched)

5. 1 and 2 and 3 and 4
Journal of Pain Research

\section{Publish your work in this journal}

The Journal of Pain Research is an international, peer reviewed, open access, online journal that welcomes laboratory and clinical findings in the fields of pain research and the prevention and management of pain. Original research, reviews, symposium reports, hypothesis formation and commentaries are all considered for publication.

\section{Dovepress}

The manuscript management system is completely online and includes a very quick and fair peer-review system, which is all easy to use. Visit http://www.dovepress.com/testimonials.php to read real quotes from published authors. 